\title{
Study on the Development of Water Quality Assessment
}

\author{
Yuan Meng ${ }^{1}$, Sudi GAO ${ }^{2}$, Xiang Shi ${ }^{3}$ \\ 1Jinhua Polytechnic: School of Architectural Engineering Institute, China \\ ${ }^{2}$ Anhui Xinhua University : School of Civil and Environmental Engineering, China \\ ${ }^{3}$ The Environmental Monitoring Station of JinHua, China
}

\begin{abstract}
KEYWORD: The Water Quality ; The Water Environment; Fuzzy Theory; Artificial Neural Network ABSTRACT: Throughout the development process of the water quality evaluation method, the paper outlines several water quality evaluation methods, summarizes them and points out the advantages and disadvantages. Finally the paper points out that water quality evaluation method will be further improved with the wide application of computer technology combined with various subjects of mutual cross-penetration.

Water quality assessment evaluates qualitatively or quantitatively the water quality elements on some water environment monitoring data and in accordance with the evaluation criteria and evaluation methods. The water quality assessment is the basis for the calculation of the capacity of the water environment and water resources planning and management. It is also a prerequisite for the water control planning. Water quality assessment results can determine the water quality model and provide parameters and information for model identification and parameter identification[1]. It can accurately reflect the water quality situation, understand and master the water pollution impact and development trends and provide a scientific basis for the protection of the water environment and water resources planning and management. Water quality assessment is not only the water quality of the scientific description of reality, but also points out the objectives and direction for water pollution control programs. We can build the appropriate water quality model for water pollution control programs by the information provided by the water quality assessment.

In the 1960s, Jacobs proposed the water quality index of concepts and formulas of the water quality evaluation. Because it is simple and easy to operate, until today people still use it in the domestic water quality evaluation practice. Later, water quality experts at home and abroad also raised various comprehensive water quality index methods. Which the Nemerow mode, mean square error mode[2] and vector mode[3] are more typical. Since the 1980s, Some uncertainty, the emergence of the nonlinear theory method and the promotion and application of computer technology provides a theoretical basis and technical support for the new model of water quality assessment[4]. Some of the commonly used methods can be summarized into the following categories throughout the development process of the water quality evaluation method.
\end{abstract}

\section{Index Evaluation Method}

Index evaluation method can be divided into single-factor evaluation and comprehensive evaluation method according to the number of evaluation projects selected by the monitoring data. Single factor evaluation method is also known as "single-indicator evaluation method" and "one vote veto method". Index evaluation is an evaluation method based on monitoring data of the physical and chemical parameters of water quality, monitoring data divided by the evaluation criteria data results the sub-indices, the water pollution index can be obtained through mathematical computation and we can use it as a water quality evaluation scale. The standard index is a representative of the single factor evaluation methods, it can be clearly pointed out where the problem is water quality but the shortcoming is that the quality status of water environment can not be given. In order to overcome the deficiencies of the method, domestic and international water quality expert propose various comprehensive water quality index methods, among them, the Nemerow index method and the weightedtype method are more typical[1].

Nemerow index method which is proposed by U.S. scholars Nemerow. Now this method is used more often.It is a balanced extreme value or highlight the maximum weighted multi-factor environmental quality index. The Nemerow Index specifically considers the most polluted factor and avoids the influence of subjective factors in the weight coefficient. 


\section{Fuzzy Theory Evaluation Method}

Because of the vagueness and the randomness of the river water environment systems, fuzzy theory evaluation method classifies objective things through the establishment of fuzzy similarity relations. In the classification of water quality assessment, research methods commonly use the clustering analysis based on equivalent fuzzy relations or fuzzy graph theory tree clustering method[5,6,7,]. The improved fuzzy hybrid cluster analysis method has been successfully used to finish the lower reach's water quality assessment of the Fengman hydropower station. Basing on fuzzy mathematics and fuzzy relation composite theory, fuzzy comprehensive evaluation quantifies some ill-defined, difficult to quantitative factors, and thus to achieve a comprehensive evaluation. In the fuzzy evaluation methods, fuzzy pattern recognition method is a fuzzy set theory's evaluation method of which develops rapidly in the field of environmental assessment in recent years [8,9]. At present, this method has been widely used in various types of water quality assessment.

With the large-scale application of gray theory in environmental systems, the gray relational analysis method of the evaluation of water quality is used. The concept of Gray relational analysis is clear and the evaluation method is convenient and operable. But its evaluation of the value tends to be homogenized, the resolution is not high. For this shortfall, on this basis, by calculating the conditional extremum, the eutrophication gray evaluation model, the water quality assessment of gray pattern recognition model, the water quality assessment of gray situation decision-making model and the water quality assessment of gray relation projection model are established.

\section{Mathematical Statistics Evaluation Method}

In recent years, constantly domestic researchers use mathematical statistics methods to evaluate water quality conditions. However, mathematical statistics often requires a large amount of measured data to support the result, it does not apply to the smaller capacity situation of the water samples.

The 25 surface water quality conditions was evaluated by using principal component analysis method. Rivers and lakes water quality was evaluated by using profiling techniques method. To promote and deepen the principal component analysis, factor analysis method also joined the water quality assessment[10]. In the comprehensive assessment of water pollution situation of the Huangshui river valley, several statistical methods were synthetically used[11]. First using the factor analysis method to evaluate, sort, and analyze the degree of water pollution of the water to be evaluated, studying each section of water pollution similarity by using cluster analysis, then the classification processing results were obtained.

\section{Artificial Neural Network Analysis Method}

The artificial neural network is a non-linear science which rises suddenly in the 1980s. In the environmental assessment, common methods in the evaluation study for river water quality and Lake water eutrophication are feedforward BP neural network model based on error back propagation algorithm and the feedback neural network model with association function-Hopfield model[12 16]. It tries to simulate the human brain's characteristics, such as self-adaptability, self-organization and fault tolerance, a better effect has been got in the field of medical diagnostics and automatic control by Analyzing and judging things. For the standard BP algorithm is slow convergence, poor stability, and easy to fall into local minima and other shortcomings, the multi-layer feedforward BP neural network constructed with radial basis function (RBF) [17] was used to evaluate the water quality and achieved good results. In addition, the combination of artificial neural networks and fuzzy mathematical theory may constitute a fuzzy neural network model of the lake water eutrophication assessment. The artificial neural network simulates the human way of thinking and intuitively infers the judgment simply the essential characteristics of things.

\section{Summary}

Rivers, lakes and other surface water bodies and groundwater is constantly contaminated with the increase of our population, economic development, and the accelerating process of urbanization. Pollution control of rivers has attracted wide attention. Water quality evaluation plays an important 
role in the prevention of water pollution. Its requirements are getting higher and higher. With the development of science and technology and the wide application of computer technology, combined with various subjects of mutual cross-penetration, water quality evaluation method will be further improved and innovative.

\section{References:}

[1] Peng Wenqi. Theories and methods of modern water quality evaluation. Beijing: Chemical Industry Press, 2005,33 39.(in Chinese)

[2] Xu Hongkai. Half set of variance of water quality evaluation model. Environmental Science, 1985, 6(4): 55 57(in Chinese)

[3] Shen Baocheng, Xu Guoyi. Hilbert space theory in the comprehensive evaluation of the environmental pollution. Environmental Science,1981 ,2(4):57 60(in Chinese)

[4] Li Ruzhong. Progress and trend analysis of theoretical models of water quality assessment. Journal of Hefei University of Technology, 2005,28(4):369 373(in Chinese)

[5] Li Xiangzhong. The application of cluster analysis in the classification of water quality. Fuzzy Mathematics, 1984,(1):85 90(in Chinese)

[6] Fu Yanpeng, GaoJiarui. Fuzzy Mathematics in the Water Quality Assessment. Wuhan: Huazhong Institute of Technology Press, 1986:35 70(in Chinese)

[7] Bai Ximeng, Man Chunsheng. Fuzzy graph tree clustering method in the evaluation of environmental quality. China Environmental Science, 1985,5(6):38 42(in Chinese)

[8] Chen Shouyi, Xiong Deqi. Fuzzy set theory and model of lake eutrophication. Journal of Lake Sciences, 1993,5(2):144 152(in Chinese)

[9] ChenSY, XiongDQ. Fuzzy set theories and methods for municipal environmental quality assessment[A].Proceeding of International Conference on City Development[C].Beijing: China Environmental Science Press, 1993:24 30

[10]Chen Dongjing,Ma Anqing,Xu Dongmin. Factor analysis in water quality assessment. Hydrological ,2002,22(3):29 31(in Chinese)

[11] Wang Xiaopeng. The application of multivariate statistical analysis in the evaluation of river pollution.Systems Engineering Theory and Practice, 2001,21(9):118 123(in Chinese)

[12] Li Zuoyong. Quality and nutritional status evaluation based on BP network model and the effect of test.Acta Scientiae Circustantiae, 1995,15(2):186 191(in Chinese)

[13]Cai Yidong,Wang Lie. Artificial neural network decision-making model of water eutrophication. China Environmental Science, 1995,15(2):123 127(in Chinese)

[14]Wang Liguan,Jia Mingtao. The neural network method of water quality evaluation and prediction. Environmental Engineering, 1998,16(2):62 65(in Chinese)

[15]Liu Guodong,Huang Chuanyou. The artificial neural network model of comprehensive evaluation of water quality. China Environmental Science, 1998,18(6):514 517(in Chinese)

[16] WalleyWJ,FonamaVN.Neural network predictors of average score pertaxon and number off amilie sat unpol-luted river sites in GreatBritain[J].Water Research,1998,32(3):613-622.

[17]Dong Manling,Huang Shengwei. Radial basis function neural networks in water quality assessment. Environmental Science and Technology,2003,26(1):23 25(in Chinese) 